\title{
New data on the fungus gnats (Diptera: Bolitophilidae, Keroplatidae, Mycetophilidae) of the Taimyr Peninsula with a description of two new species of the genus Boletina Staeger
}

\section{Новые данные по фауне грибных комаров (Diptera: Bolitophilidae, Keroplatidae, Mycetophilidae) полуострова Таймыр с описанием двух новых видов рода Boletina Staeger}

\author{
A.V. Polevoi ${ }^{1}$, Yu.V. Maximova ${ }^{2}$, E.Yu. Subbotina ${ }^{2}$ \\ А.В. Полевой ${ }^{1}$, Ю.В. Максимова ${ }^{2}$, Е.Ю. Субботина ${ }^{2}$
}

\footnotetext{
${ }^{1}$ Forest Research Institute of Karelian Reserach Centre of Russian Academy of Sciences, Pushkinskaya 11, Petrozavodsk 185910, Russia. E-mail: alexei.polevoi@krc.karelia.ru.

Институт леса Карельского Научного центра РАН, Пушкинская 11, 185910 Петрозаводск, Россия.

2 Tomsk State University, Lenina Prospekt 36, Tomsk 634050, Russia. E-mail: allodia@sibmail.com; elena.y.subbotina@mail.ru.

2 Томский государственный университет, просп. Ленина 36, 634050 Томск, Россия.
}

KEY WORDS: fungus gnats, fauna, new species, Taimyr, Russia.

КЛЮЧЕВЫЕ СЛОВА: грибные комары, фауна, новые виды, Таймыр, Россия.

ABSTRACT. New data on the fungus gnats fauna of the Taimyr Peninsula (Russia) are presented. Seventytwo species of the families Bolitophilidae, Keroplatidae and Mycetophilidae are reported, 65 of which are new for the area. Five species are newly recorded from Russia and two species - from the Palaearctic region. Two new species: Boletina rodentistyla Polevoi, sp.n. and Boletina pyasina Maximova et Subbotina, sp.n. are described.

РЕЗЮМЕ. Представлены новые данные по фауне грибных комаров полуострова Таймыр (Россия). Аннотированный список включает 72 вида из семейств Bolitophilidae, Keroplatidae и Mycetophilidae, 65 из которых впервые зарегистрированы в регионе. Пять видов впервые зарегистрированы на территории России, два вида - впервые в палеарктическом регионе. Приводятся описания двух новых видов: Boletina rodentistyla Polevoi, sp.n. и Boletina pyasina Maximova et Subbotina, sp.n.

\section{Introduction}

The fauna of fungus gnats in Russia is comparatively well studied in general, however, the knowledge on different parts of its territory is highly unequal. Taimyr peninsula is among the regions with very little data available. Three species were reported from the west Taimyr at the beginning of the XX century [Lundsröm, 1915], and the only later addition includes seven species of the genera Sciophila Meigen, 1818, Boletina Staeger, 1840, Coelosia Winnertz, 1863 and Gnoriste Meigen, 1818 [Zaitzev, 1994]. During recent years we can observe a growing interest of entomologists to the northern areas of Russia. As a result, significant new materials were obtained from subarctic and arctic zones. In this paper, we present new data on the fungus gnats of the Taimyr peninsula. The list includes 72 species, 65 of which are new for the area. Five species are newly recorded from Russia and two species - from the Palaearctic region. Two species of the genus Boletina are described as new for science.

\section{Study area}

The study area lies in the limits of the subarctic permafrost zone and characterized by excessive humidity. It comprises four localities in the west part of the Taimyr Peninsula (Fig. 1), which administratively belongs to the Dolgano-Nenetskiy Autonomous District of the Krasnoyarsk Region of Russia. Details on each locality, including brief information on habitats and collecting methods, are given below.

1. The Lemberov River, $12.5 \mathrm{~km} \mathrm{~S}$ of Dikson, $73.40^{\circ} \mathrm{N}, 80.65^{\circ} \mathrm{E}$ (Lemberov in the species list). River bank. Yellow pan traps. Collected by A. Barkalov.

2. Right bank of the River Pyasina, near the River Tareya, $73.26^{\circ} \mathrm{N}, 90.59^{\circ} \mathrm{E}$ (Tareya). Zonal tundra communities, river banks, river-side ravines. Pitfall traps and yellow pan traps. Collected by O. Makarova and A. Babenko.

3. Nyapan Ridge, $9 \mathrm{~km}$ NW of the Lake Pyasino, near the Lake Ladannah, $70.08^{\circ} \mathrm{N}, 87.50^{\circ} \mathrm{E}$ (Ladannah). Sedgedwarf tundra communities, sedge mires, open Larix forest. Pitfall traps. Collected by O. Makarova.

How to cite this article: Polevoi A.V., Maximova Yu.V., Subbotina E.Yu. 2020. New data on the fungus gnats (Diptera: Bolitophilidae, Keroplatidae, Mycetophilidae) of the Taimyr Peninsula with a description of two new species of the genus Boletina Staeger// Russian Entomol. J. Vol.29. No.3. P.315-326. doi: 10.15298/rusentj.29.3.13 


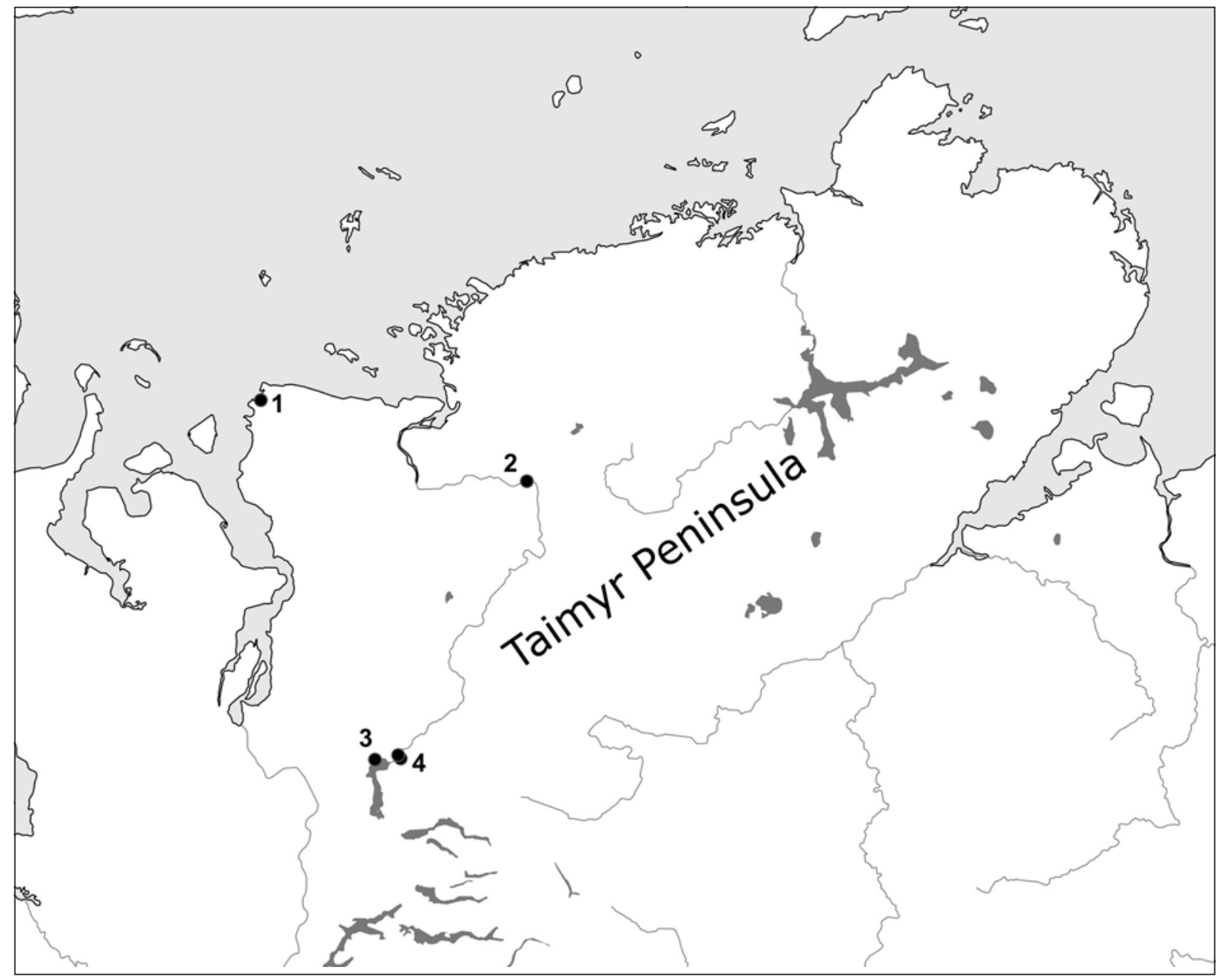

Fig. 1. Collecting localities: 1 - the Lemberov River, $12,5 \mathrm{~km} \mathrm{~S}$ of Dikson; 2 - Right bank of the River Pyasina, near the River Tareya; 3 - Nyapan Ridge, $9 \mathrm{~km} \mathrm{NW}$ of the Lake Pyasino, near the Lake Ladannah; 4 - the River Pyasina, 90-93 km N of Norilsk.

Рис. 1. Точки сборов: 1 - река Лемберова, 12,5 км южнее пос. Диксон; 2 - правый берег реки Пясина, рядом с устьем р. Тарея; 3 - гряда Няпань, 9 км северо-восточнее оз. Пясино, рядом с оз. Ладаннах; 4 - река Пясина, 90-93 км севернее Норильска.

4. The River Pyasina, $90-93 \mathrm{~km} \mathrm{~N}$ of Norilsk, $70.15^{\circ} \mathrm{N}$, $88.28^{\circ} \mathrm{E} ; 70.18^{\circ} \mathrm{N}, 88.18^{\circ} \mathrm{E}$ (Pyasina). Sedge-dwarf tundra communities and open boreal woodland. Malaise trap and sweep-netting. Collected by M. Shcherbakov.

\section{Methods}

All materials were initially kept in $70 \%$ or $96 \%$ alcohol. After identification, the specimens were partly pinned together with dissected terminalia in glycerine filled micro vials. $Z$-stacked images of male genitalia were obtained with a Leica DM1000 microscope equipped with LOMO MC-60 digital camera. Images were combined using Helicon Focus software (version 7.5.4). The morphological terminology follows Söli [1997a], except for wing venation, which is given following newer interpretation [Saigusa, 2006; Amorim, Rindal, 2007; Borkent, Wheeler, 2012]. Species are listed in alphabetic order inside higher taxonomic groups, which order follows Kjærandsen et al. [2007a].
Materials are stored in the following collections: FRIP — Forest Research Institute (Petrozavodsk, Russia) ISEA - Institute of Systematics and Ecology of Animals, Siberian Branch of the Russian

Academy of Sciences (Novosibirsk, Russia)

UTR - Tomsk State University (Tomsk, Russia)

ZISP — Zoological Institute (Saint-Petersburg, Russia)

Species list

$$
\text { Family Bolitophilidae }
$$

Bolitophila (Bolitophila) cinerea Meigen, 1818

MATERIAL. $1 \sigma^{7}$, Tareya, 2-7.08.2010 (FRIP); 97 ○ $^{7}, 2$ ㅇ, Lemberov, 19.07.2012 (ISEA).

DISTRIBUTION. Holarctic: Greenland [Söli et al., 2015], widely distributed in the Palaearctic region [Zaitzev, 1999, Kjærandsen et al., 2007a].

Bolitophila (Cliopisa) hybrida (Meigen, 1804)

MATERIAL. $20^{7} \sigma^{7}$, Pyasina, 9-13.08.2015 (UTR).

DISTRIBUTION. Palaearctic, widely distributed [Zaitzev, 1999, Kjærandsen et al., 2007a]. 
Bolitophila (Cliopisa) rossica Landrock, 1912

MATERIAL. 8 o $^{7}$, Pyasina, 9-13.08.2015 (UTR).

DISTRIBUTION. Palaearctic, widely distributed [Zaitzev, 1999, Kjærandsen et al., 2007a].

\section{Family Keroplatidae}

Subfamily Keroplatinae

Tribe Orfeliini

Urytalpa nigrita (Johannsen, 1910)

MATERIAL. 4 or $0^{7}$, Ladannah, 4-15.07.1999 (FRIP).

DISTRIBUTION. Nearctic, known by two specimens from USA and Canada [Kjærandsen et al., 2009]. Here for the first time recorded from the Palaearctic region and Russia.

REMARKS. Taimyr specimens (Fig. 2) have minor differences in the basal part of the male aedeagal apparatus (e.g. presence of additional basal appendages), comparing with Canadian specimen, figured by Kjærandsen et al. [2009]. $\mathrm{We}$, however, believe they are conspecific, considering the general similarity of terminalia and especially characteristic apex of aedeagus, forming four small projections [cf. Kjærandsen et al. 2009, p. 45, Fig. 9 A]. There are 3 other specimens in the series with slightly different structure of male terminalia (Fig. 3), and probably representing distinct species somewhat more similar to U. rhapsodica Chandler, 1995.

\section{Family Mycetophilidae \\ Subfamily Mycomyinae}

Mycomya (Mycomya) denmax Väisänen, 1979

MATERIAL. $2 \sigma^{7} \sigma^{7}$, Pyasina, 9-13.08.2015 (UTR).

DISTRIBUTION. Holarctic, widely distributed [Väisänen, 1984; Kjærandsen et al., 2007a; Zaitzev, 1999].

\section{Mycomya (Mycomya) fornicata (Lundström, 1911)}

MATERIAL. $20^{7} \sigma^{7}$, Pyasina, 9-13.08.2015 (UTR).

DISTRIBUTION. Palaearctic: European Alps, NW and E Russia [Väisänen, 1984; Zaitzev, 1999].
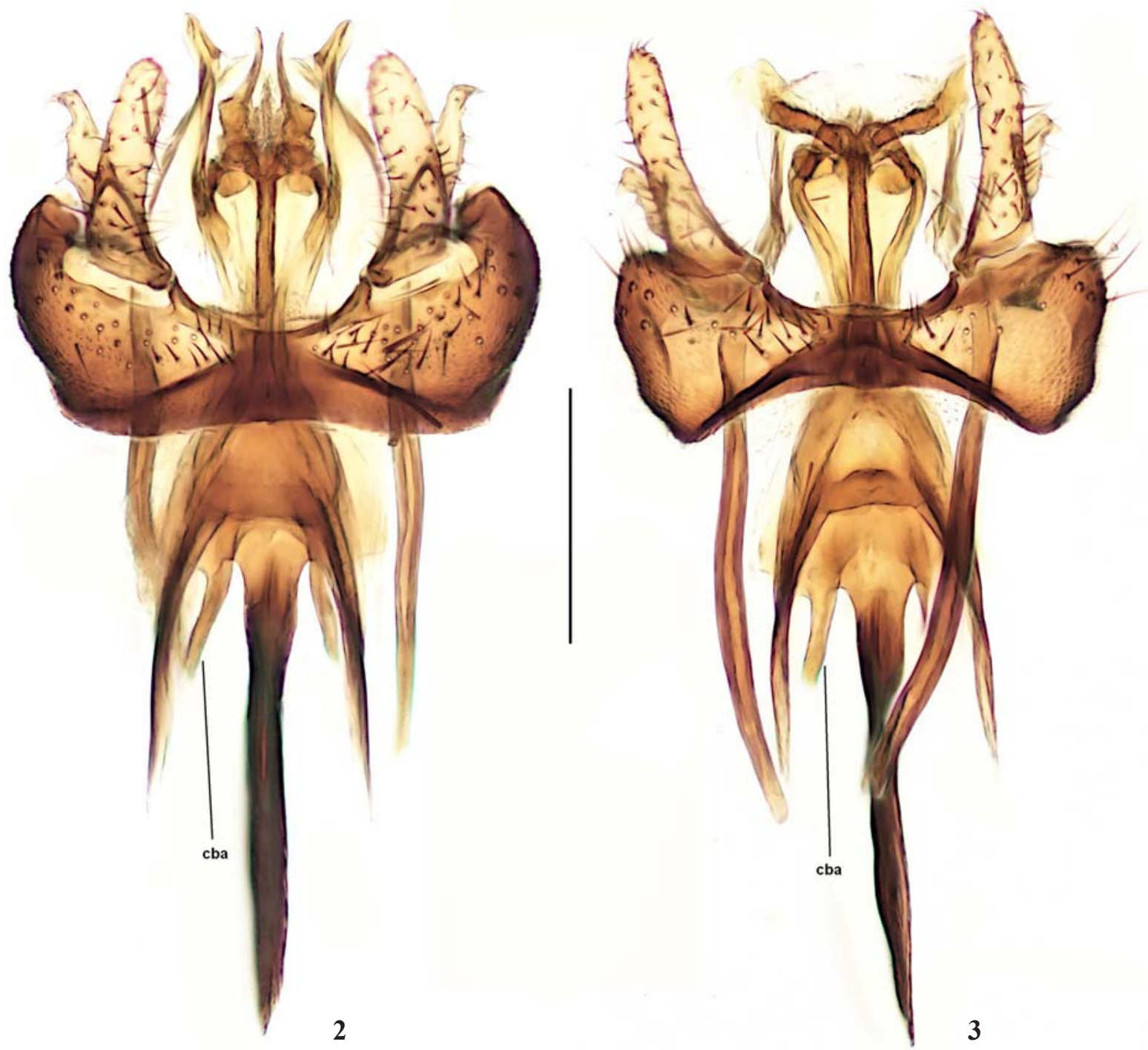

Figs 2-3. Urytalpa spp., male terminalia, ventral view: 2 - Urytalpa nigrita; 3 - unrecognized species. Scale bar: $0.2 \mathrm{~mm}$. Abbreviation: cba - complementary basal appendages.

Рис. 2-3. Гениталии самцов Urytalpa spp., с вентральной стороны: 2 - Urytalpa nigrita; 3 - неописанный вид. Масштаб: 0,2 $\mathrm{mm}$. Сокращения: cba - дополнительные базальные отростки. 
Mycomya (Mycomya) humida Garrett, 1924

MATERIAL. $10^{7}$, Pyasina, 9-13.08.2015 (UTR).

DISTRIBUTION. Holarctic: USA, Canada, Russia (Karelia and Ural), Finland, Sweden, Norway [Väisänen, 1984; Kjærandsen et al., 2007a]. Here for the first time recorded from the Asian part of the Palaearctic region.

Mycomya (Mycomya) islandica Väisänen, 1984

MATERIAL. 1 O$^{7}$, Ladannah, 4-15.07.1999 (FRIP).

DISTRIBUTION. Holarctic, known from northern localities [Väisänen, 1984; Kjærandsen et al., 2007a; Söli et al., 2015]. In Russia was recorded from Murmansk Province [Väisänen, 1984] and N Karelia [Jakovlev et al., 2014].

Mycomya (Mycomya) neohyalinata Väisänen, 1984

MATERIAL. $10^{7}$, Pyasina, 9-13.08.2015 (UTR).

DISTRIBUTION. Holarctic: USA, Canada, Europe. In Russia recorded from European part to Siberia. [Väisänen, 1984; Zaitzev, 1994].

\section{Subfamily Sciophilinae}

Acnemia angusta Zaitzev, 1982

MATERIAL. $1 O^{7}$, Pyasina, 9-13.08.2015 (UTR).

DISTRIBUTION. Europe: NW Russia (Karelia and Leningrad Province), Finland, Sweden, and Bulgaria. [Zaitzev, 1994; Kjærandsen et al., 2007a]. Here for the first time recorded from the Asian part of the Palaearctic region.

\section{Acnemia falcata Zaitzev, 1982}

MATERIAL. $10^{7}$, Pyasina, 9-13.08.2015 (UTR).

DISTRIBUTION. Palaearctic: NW Russia (Karelia and Murmansk Province), Finland, Sweden, Norway, Czech Republic, and Bulgaria. [Zaitzev, 1994; Kjærandsen et al., 2007a].

REMARKS. East Palaearctic record in the Fauna Europaea online database is erroneous (P. Chandler, pers. comm). The new record is hence the first one confirming the occurrence of A. falcata in the Asian part of the Palaearctic region.

\section{Sciophila fuliginosa Holmgren, 1883}

MATERIAL. 3 O $^{7} \sigma^{\top}$, Lemberov, 19.07.2012 (ISEA)

DISTRIBUTION. Holarctic: USA, Canada, Finland, Russia (Novaya Zemlya and Taimyr peninsula). Limited to arctic zone [Jakovlev et al., 2014].

\section{Sciophila hirta Meigen, 1818}

MATERIAL. 1 or $^{7}$, Ladannah, 6-14.07.1999 (FRIP).

DISTRIBUTION. Holarctic. Detailed distribution is unclear due to questionable identity of older records [Kjærandsen et al., 2007a].

REMARKS. Closely related species $S$. parviareolata Santos Abreu, 1920 has been reported from N Europe [Kjaerandsen et al., 2007b]. We have examined several other specimens from N Russia, which could not be clearly recognized either as $S$. hirta or as $S$. parviareolata using characters indicated by Chandler [2001]. It was pointed earlier that the validity of $S$. parviareolata is questionable [Kjaerandsen et al., 2007b], though there may be additional species in this complex.

\section{Sciophila longua Garrett, 1925}

MATERIAL. 49 O $^{7}$, Lemberov, 19.07.2012 (ISEA).

DISTRIBUTION. Nearctic: Canada [Zaitzev 1982]. Here for the first time recorded from the Palaearctic region and Russia.

Sciophila vakulenkoi Stackelberg, 1943

MATERIAL. 51 o $^{\top}$, Lemberov, 19.07.2012 (ISEA).
DISTRIBUTION. Holarctic: Russia (Novaya Zemlya) and Canada [Zaitzev, 1982].

\section{Subfamily Gnoristinae}

Boletina arctica Holmgren, 1872

MATERIAL. $2 O^{7} \sigma^{7}$, Pyasina, 9-13.08.2015 (UTR).

DISTRIBUTION. Holarctic: Arctic Russia (Novaya Zemlya and Dikson Isl.), Canada and Greenland [Zaitzev 1994, Salmela et al., 2016].

\section{Boletina birulai Lundström, 1915}

MATERIAL. $9 \sigma^{7} \sigma^{7}, 1$ 9 , Lemberov, 19.07.2012 (ISEA); 6 $0^{7} \sigma^{7}, 41$ 우, Ladannah, 4-15.07.1999 (FRIP).

DISTRIBUTION. Holarctic: USA, Canada, Russia [Salmela et al., 2016]. In Russia was recorded from Dikson Isl. and New-Siberian Isl. [Lundsröm, 1915; Zaitzev, 1994].

REMARKS. There are records from Germany and Poland in the Fauna Europaea online database, however, according to Salmela et al. [2016], these are erroneous or need verification.

Boletina borealis Zetterstedt, 1852

MATERIAL. $10 \mathrm{O}^{7} \mathrm{O}^{7}$, Lemberov, 19.07.2012 (ISEA); $6 \mathrm{O}^{7} \mathrm{O}^{7}, 8$ of, Ladannah, 4-15.07.1999 (FRIP).

DISTRIBUTION. Palaearctic: from Europe to Japan [Zaitzev, 1999; Jakovlev et al., 2014].

\section{Boletina dubia (Meigen, 1804)}

MATERIAL. $20^{7} \sigma^{7}$, Ladannah, 8-15.07.1999 (FRIP).

DISTRIBUTION. Europe, widely distributed [Jakovlev et al., 2014]. In Russia was known from Leningrad Province [Zaitzev, 1994]. Here for the first time recorded from the Asian part of the Palaearctic region.

Boletina gripha Dziedzicki, 1885

MATERIAL. $3 \mathrm{O}^{7} \sigma^{\top}$, Pyasina, 9-13.08.2015 (UTR).

DISTRIBUTION. Palaearctic, widely distributed [Zaitzev, 1999; Kjærandsen et al., 2007a].

Boletina hedstroemi Polevoi et Hedmark, 2004

MATERIAL. $30^{7} \bigcirc^{7}$, Pyasina, 9-13.08.2015 (UTR).

DISTRIBUTION. Europe: NW Russia (Karelia and Murmansk Province), Finland, Sweden [Kjærandsen et al., 2007a; Polevoi, 2010]. Here for the first time recorded from the Asian part of the Palaearctic region.

\section{Boletina hyperborea Salmela, 2016}

MATERIAL. 2 O$^{7} \sigma^{7}, 11$ 우, Ladannah, 4-15.07.1999 (FRIP).

DISTRIBUTION. Holarctic: Canada, Finland, Norway, and Sweden [Salmela et al., 2016]. Here for the first time recorded from the Asian part of the Palaearctic region and Russia.

Boletina lapponica Polevoi et Hedmark, 2004

MATERIAL. 25 o $^{7} \sigma^{7}$, Pyasina, 9-13.08.2015 (UTR).

DISTRIBUTION. Europe: NW Russia (Karelia), Finland, and Sweden [Jakovlev et al., 2014]. Here for the first time recorded from the Asian part of the Palaearctic region.

Boletina onegensis Polevoi, 1995

MATERIAL. $1 \sigma^{7}$, Pyasina, 9-13.08.2015 (UTR).

DISTRIBUTION. Palaearctic: N Russia (Karelia, Murmansk Province), Finland and Sweden [Kjærandsen et al., 2007a; Polevoi, 2010].

REMARKS. Kjærandsen et al. [2007a] pointed out that B. takagii Sasakawa et Kimura, 1974 in the sense of Zaitzev [1994] is in fact B. onegensis. This statement was probably 
based on the terminalia figures [Zaitzev 1994, p. 228, Fig. 9, $11]$ which indeed show some similarity with $B$. onegensis. However, the male specimen of B. takagii from Sakhalin, stored in Moscow (examined by the first author) represents true $B$. takagii, agreeing well with the description and figures by Sasakawa and Kimura [1974]. The new record is hence the first one confirming the occurrence of $B$. onegensis in the Asian part of the Palaearctic region.

\section{Boletina palmata Polevoi, 2013}

MATERIAL. 3 o $^{7}$, Pyasina, 9-13.08.2015 (UTR).

DISTRIBUTION. Palaearctic: Russia (Karelia, Murmansk Province, and Chukotka), Finland, Norway, Sweden [Jakovlev et al., 2014; Polevoi, Barkalov, 2017].

Boletina sciarina Staeger, 1840

MATERIAL. $1 \bigcirc^{7}$, Pyasina, 9-13.08.2015 (UTR).

DISTRIBUTION. Holarctic, widely distributed [Zaitzev, 1999; Kjærandsen et al., 2007a].

Boletina tiroliensis Plassmann, 1980

MATERIAL. 18 O $^{7}$ O $^{\top}$, Pyasina, 9-13.08.2015 (UTR).

DISTRIBUTION. Palaearctic: N Russia (from Murmansk Province and Karelia to Yakutia), Fennoscandia, and Austria [Jakovlev et al., 2014].

\section{Coelosia truncata Lundström, 1909}

MATERIAL. $1 \sigma^{7}$, Tareya, 20.07-8.08.2010 (FRIP), $1 \sigma^{7}$, Pyasina, 9-13.08.2015 (UTR).

DISTRIBUTION. Holarctic, widely distributed [Söli, 1997b].

REMARKS. Older records of C. truncata from the Palaearctic region need verification to avoid confusion with closely related C. gracilis Johannsen, 1912, which has been recently discovered in Finland [Jakovlev et al., 2014].

\section{Coelosia tundrica Zaitzev, 1994}

MATERIAL. 3 o $^{7}$, Pyasina, 9-13.08.2015 (UTR).

DISTRIBUTION. E Siberia, so far recorded only from Dikson Isl. [Zaitzev, 1994].

Katatopygia erythropyga (Holmgren, 1883)

MATERIAL. $10^{7}, 1$, , Lemberov, 19.07.2012 (ISEA).

DISTRIBUTION. Holarctic: NW USA and N Palaeactic [Martinsson, Kjærandsen, 2012]. In Russia was recorded from Karelia, Murmansk Province, Novaya Zemlya, Vaigach Isl., and Chukotka [Holmgren, 1883; Zaitzev, Polevoi, 2001; Polevoi, 2010; Polevoi, Barkalov, 2017].

REMARKS. Some older records of this species (including those from the E Palaearctic are questionable due to possible confusion with $K$. sahlbergi (Lundström, 1906) [Zaitzev, Polevoi, 2001].

\section{Subfamily Mycetophilinae \\ Tribe Exechiini}

Allodia (Allodia) embla Hackman, 1971

MATERIAL. $15 O^{7} \sigma^{7}$, Ladannah, 4-15.07.1999 (FRIP).

DISTRIBUTION. Holarctic: USA, Canada [Zaitzev, 1983); NW Europe [Kjærandsen et al., 2007a); Karelia and Chukotka in Russia [Polevoi, 2000; Polevoi, Barkalov, 2017].

Allodia (Allodia) septentrionalis Hackman, 1971

MATERIAL. $13 \sigma^{7} \sigma^{7}$, Lemberov, 19.07.2012 (ISEA); $7 \sigma^{7} \sigma^{7}$, Ladannah, 4-15.07.1999 (FRIP).

DISTRIBUTION. Palaearctic, widely distributed [Zaitzev, 1983; Kjærandsen et al., 2007a].
Allodia (Allodia) tuomikoskii Hackman, 1971

MATERIAL. 1 o', Lemberov, 19.07.2012 (ISEA).

DISTRIBUTION. Holarctic: USA [Zaitzev, 1983]; Europe and E Russia [Zaitzev, 2003; Polevoi, Barkalov, 2017].

Allodia (Brachycampta) subpistillata Ševčík, 1999

MATERIAL. 1 O’, Ladannah, 4-15.07.1999 (FRIP).

DISTRIBUTION. Europe: Finland, NW Russia (Karelia), Sweden, and Czech Republic [Jakovlev et al., 2014].

Anatella ciliata Winnertz, 1863

MATERIAL. $1 \sigma^{7}$, Ladannah, 4-15.07.1999

DISTRIBUTION. Holarctic, widely distributed [Zaitzev, 2003; Kjærandsen et al., 2007a].

Brevicornu arcticoides Caspers, 1985

MATERIAL. $1 \sigma^{7}$, Tareya, 20.07-8.08.2010, (FRIP); $10 \sigma^{7} \sigma^{7}$, Lemberov, 19.07.2012(ISEA); $6 \sigma^{7} \sigma^{\top}$, Ladannah, 4-15.07.1999(FRIP).

DISTRIBUTION. Europe: NW part [Kjærandsen et al., 2007a], Baltia, Transcaucasia, European part of Russia [Zaitzev, 2003]. Here for the first recorded from the Asian part of the Palaearctic region.

Brevicornu auriculatum (Edwards, 1925)

MATERIAL. $10^{7}$, Tareya, 20.07-8.08.2010, (FRIP); 5 O $^{7} 0^{7}$, Lemberov, 19.07.2012 (ISEA)

DISTRIBUTION. Palaearctic: W Europe and Russian Far East [Jakovlev et al., 2014].

Brevicornu bipartitum Laštovka et Matile, 1974

MATERIAL. $1 \sigma^{7}$, Tareya, 20.07-8.08.2010, (FRIP); $22 \sigma^{7} \sigma^{7}$, Lemberov, 19.07.2012 (ISEA).

DISTRIBUTION. Holarctic: USA [Zaitzev, 1988); Fennoscandia, NW and E Russia, Mongolia [Zaitzev, 2003; Kjærandsen et al., 2007a; Polevoi, Barkalov, 2017].

Brevicornu cognatum Ostroverkhova, 1979

MATERIAL. 7 O$^{7} \sigma^{7}$, Lemberov, 19.07.2012 (ISEA).

DISTRIBUTION. Holarctic: USA [Zaitzev, 1985], Sweden and Finland [Jakovlev et al., 2014], N Caucasus and E Russia [Zaitzev, 2003].

Brevicornu griseolum (Zetterstedt, 1852)

MATERIAL. $9 \sigma^{7} \sigma^{7}$, Lemberov, 19.07.2012 (ISEA).

DISTRIBUTION. Holarctic: Greenland [Söli et al., 2015], NW Europe and mountainous regions in the south [Kjærandsen et al., 2007a]. In Russia recorded from Leningrad Province, Karelia, and Murmansk Province [Krivosheina et al., 1986; Zaitzev, 2003, as B. boreale Lundström].

Brevicornu proximum (Staeger, 1840)

MATERIAL. 2 O $^{7}$, Ladannah, 5-14.07.1999 (FRIP).

DISTRIBUTION. Palaearctic: from Europe to Siberia [Zaitzev, 1985; Kjærandsen et al., 2007a].

Cordyla fusca Meigen, 1804

MATERIAL. $1 \sigma^{7}$, Pyasina, 9-13.08.2015 (UTR).

DISTRIBUTION. Palaearctic: from Europe to Siberia [Zaitzev, 2003; Kjærandsen et al., 2007a)

Cordyla murina Winnertz, 1863

MATERIAL. $1 \sigma^{7}$, Pyasina, 9-13.08.2015 (UTR).

DISTRIBUTION. Palaearctic, widely distributed [Zaitzev, 2003; Kjærandsen et al., 2007a].

Cordyla semiflafa (Staeger, 1840)

MATERIAL. $2 \sigma^{7} \sigma^{7}$, Pyasina, 9-13.08.2015 (UTR). 
DISTRIBUTION. Palaearctic, widely distributed [Zaitzev, 2003; Kjærandsen et al., 2007a].

Exechia cincta Winnertz, 1863

MATERIAL. 2 O$^{7} \sigma^{7}$, Pyasina, 9-13.08.2015 (UTR).

DISTRIBUTION. Palaearctic, widely distributed [Zaitzev, 2003; Kjærandsen et al., 2007a].

\section{Exechia frigida (Boheman, 1865)}

MATERIAL. 171 ○’ $^{7}$, Tareya, 20.07-8.08.2010, (FRIP); 96 $\sigma^{7} \sigma^{7}$, Lemberov, 19.07.2012 (ISEA), $12 \sigma^{7} \sigma^{7}$, Pyasina, 9-13.08.2015 (UTR).

DISTRIBUTION. Holarctic, widely distributed [Zaitzev, 2003; Kjærandsen et al., 2007b].

Exechia macula Chandler, 2001

MATERIAL. 8 or $\sigma^{7}$, Pyasina, 9-13.08.2015 (UTR).

DISTRIBUTION. Palaearctic, widely distributed [Zaitzev, 2003; Kjærandsen et al., 2007a].

Exechia micans Laštovka et Matile, 1974

MATERIAL. 5 o $\sigma^{7}$, Pyasina, 9-13.08.2015 (UTR).

DISTRIBUTION. Holarctic: Finland, Germany, Iceland, Norway, Sweden, Russia (Karelia, Murmansk Province and Chukotka), Mongolia [Jakovlev et al., 2014; Söli et al., 2015; Polevoi Barkalov, 2017].

\section{Exechia parvula (Zetterstedt, 1852)}

MATERIAL. $1 \sigma^{7}$, Pyasina, 9-13.08.2015 (UTR).

DISTRIBUTION. Palaearctic: from Europe to Siberia [Zaitzev, 2003; Kjærandsen et al., 2007a].

Exechia pseudofestiva Lackschewitz, 1937

MATERIAL. $10^{7}$, Ladannah, 8-15.07.1999 (FRIP).

DISTRIBUTION. Palaearctic: NW Europe and Russian Far East [Zaitzev, 2003; Kjærandsen et al., 2007a]

Exechia separata Lundström, 1912

MATERIAL. $1 \sigma^{7}$, Ladannah, 4-15.07.1999 (FRIP), 13 ○ $^{7}$, Pyasina, 9-13.08.2015 (UTR).

DISTRIBUTION. Holarctic, widely distributed [Zaitzev, 2003; Kjærandsen et al., 2007a].

Exechia similis Laštovka et Matile, 1974

MATERIAL. $1 \sigma^{7}$, Lemberov, 19.07.2012 (ISEA); $5 \sigma^{7} \sigma^{7}$, Ladannah, 4-15.07.1999 (FRIP), 32 O' Oे $^{7}$, Pyasina, 9-13.08.2015 (UTR).

DISTRIBUTION. Palaearctic: Europe, W Siberia and Mongolia [Zaitzev, 2003; Kjærandsen et al., 2007a; Subbotina, 2014].

Exechia spinigera Winnertz, 1863

MATERIAL. $64 \mathrm{O}^{7} \mathrm{O}^{7}$, Tareya, 25.07-7.08.2010, (FRIP). DISTRIBUTION. Palaearctic, widely distributed [Zaitzev, 2003; Kjærandsen et al., 2007a].

REMARKS. The identity of E. spinigera is questionable due to the loss of the type material [Kjærandsen et al., 2007a]. Here we follow the interpretation by A. Zaitzev [2003].

Exechia spinuligera Lundström, 1912

MATERIAL. $330^{7} 0^{7}$, Tareya, 25.07-7.08.2010, (FRIP); 3 $\mathrm{O}^{7} \mathrm{O}^{2}$, Lemberov, 19.07.2012 (ISEA)

DISTRIBUTION. Palaearctic: Europe and Russian Far East [Zaitzev, 2003; Kjærandsen et al., 2007a].

Exechia unimaculata (Zetterstedt, 1860)

MATERIAL. $10^{7}$, Tareya, 2-7.08.2010, (FRIP).

DISTRIBUTION. Palaearctic: N Europe and E Russia [Zaitzev, 2003; Kjærandsen et al., 2007a; Polevoi, Barkalov, 2017].
Rymosia coulsoni Chandler, 1994

MATERIAL. 4 O $^{7}$, Pyasina, 9-13.08.2015 (UTR).

DISTRIBUTION. Europe, so far only known from Great Britain [Chandler, 1994]. Here for the first time recorded from the Asian part of the Palaearctic region and Russia.

Rymosia sagulata Plassmann, 1976

MATERIAL. $3 \sigma^{7} \sigma^{7}, 1$ ㅇ, Tareya, 20.07-8.08.2010, (FRIP); 36 $0^{7} \sigma^{7}$, Lemberov, 19.07.2012 (ISEA).

DISTRIBUTION. Palaearctic: E Russia (Altai Mts. and Tuva), Sweden and Slovakia [Zaitzev, 2003; Kjærandsen et al., 2007a].

Rymosia speyae Chandler, 1994

MATERIAL. $10^{7}$, Lemberov, 19.07.2012 (ISEA).

DISTRIBUTION. Europe: Great Britain and Iceland [Kjærandsen et al., 2007b]. Here for the first time recorded from the Asian part of the Palaearctic region and Russia.

Tarnania tarnanii (Dziedzicki, 1910)

MATERIAL. $5 \sigma^{7} \sigma^{7}, 1$, , Tareya, 20.07-8.08.2010 (FRIP); 11 $\sigma^{7} \sigma^{7}$, Lemberov, 19.07.2012 (ISEA), $2 O^{7} \sigma^{7}$, Pyasina, 9-13.08.2015 (UTR).

DISTRIBUTION. Holarctic, widely distributed [Kjærandsen, 2006].

Tribe Mycetophilini

Mycetophila boreocruciator Ševčík, 2003

MATERIAL. 2 O $^{7} \sigma^{7}$, Ladannah, 6-14.07.1999 (FRIP). $20^{7} \sigma^{7}$, Pyasina, 9-13.08.2015 (UTR).

DISTRIBUTION. Europe: Estonia, Finland, Norway, NW Russia (Murmansk Province), Slovakia, and Sweden [Jakovlev et al., 2014].

Mycetophila evanida Laštovka, 1972

MATERIAL. $1 \sigma^{7}$, Tareya, 2-7.08.2010, (FRIP); $1 \sigma^{7}$, Lemberov, 19.07.2012 (ISEA).

DISTRIBUTION. Holarctic: Greenland [Söli et al., 2015], Europe and Mongolia [Laštovka, 1972; Jurgenstern et al., 2015]. In Russia was recorded from Karelia [Zaitzev, 2003].

Mycetophila ichneumonea Say, 1823

MATERIAL. 3 o $^{7}$, Lemberov, 19.07.2012 (ISEA).

DISTRIBUTION. Holarctic, widely distributed [Jurgenstern et al., 2015].

Mycetophila luctuosa Meigen, 1830

MATERIAL. $2 \sigma^{7} \sigma^{7}$, Lemberov, 19.07.2012 (ISEA).

DISTRIBUTION. Holarctic, widely distributed [Zaitzev, 2003; Kjærandsen et al., 2007a].

Mycetophila riparia Chandler, 1993

MATERIAL. $7 \sigma^{7} \sigma^{7}$, Pyasina, 9-13.08.2015 (UTR).

DISTRIBUTION. Holarctic: Russia (W Siberia and Chukotka), USA [Chandler, 1993; Ostroverkhova, Maksimova, 2000; Polevoi, Barkalov, 2017].

Mycetophila sordida van der Wulp, 1874

MATERIAL. $1 \mathrm{O}^{\top}$, Pyasina, 9-13.08.2015 (UTR).

DISTRIBUTION. Holarctic: USA and Canada [Laffoon, 1956], from Europe to Siberia in the Palaearctic region [Zaitzev, 2003; Kjærandsen et al., 2007a].

Mycetophila strobli Laštovka, 1972

MATERIAL. $10^{7}$, Pyasina, 9-13.08.2015 (UTR).

DISTRIBUTION. Palaearctic, widely distributed [Zaitzev, 2003; Jurgenstern et al., 2015]. 
Mycetophila subsigillata Zaitzev, 1999

MATERIAL. $1 \sigma^{7}$, Lemberov, 19.07.2012 (ISEA).

DISTRIBUTION. Palaearctic, widely distributed [Zaitzev, 2003; Kjærandsen et al., 2007a].

REMARKS. Only scattered records are known from Europe, but the species is probably more abundant, as could be earlier mixed with M. sigillata Dziedzicki, 1884 [Kjærandsen et al., 2007a].

Phronia egregia Dziedzicki, 1889

MATERIAL. $1 \sigma^{7}$, Lemberov, 19.07.2012 (ISEA).

DISTRIBUTION. Holarctic, widely distributed [Zaitzev, 2003; Kjærandsen et al., 2007a; Söli et al., 2015].

Sceptonia demeijerei Bechev, 1997

MATERIAL. $1 \sigma^{7}$, Lemberov, 19.07.2012 (ISEA).

DISTRIBUTION. Europe: NW Russia (Karelia), Czech Republic and Slovakia, Germany, The Netherlands, Fennoscandia [Kjærandsen et al., 2007a; Kjærandsen, Jordal, 2007]. Here for the first time recorded from the Asian part of the Palaearctic region.

Sceptonia fumipes Edwards, 1925

MATERIAL. $40^{7} \sigma^{7}$, Lemberov, 19.07.2012 (ISEA).

DISTRIBUTION. Palaearctic: widely distributed in Europe [Kjærandsen et al., 2007a], NW and E Russia [Zaitzev, 2003; Polevoi, Barkalov, 2017].

Trichonta venosa (Staeger, 1840)

MATERIAL. $1 \mathrm{O}^{7}$, Lemberov, 19.07.2012 (ISEA).

DISTRIBUTION. Holarctic: widespread in N America [Gagné, 1981], in the Palaearctic region recorded from Europe to Siberia [Zaitzev, 2003; Kjærandsen et al., 2007a].

Zygomia pseudohumeralis Caspers, 1980

MATERIAL. $1 \mathrm{O}^{7}$, Pyasina, 9-13.08.2015 (UTR).

DISTRIBUTION. Palaearctic: from Europe to Siberia [Zaitzev, 2003; Kjærandsen et al., 2007a].

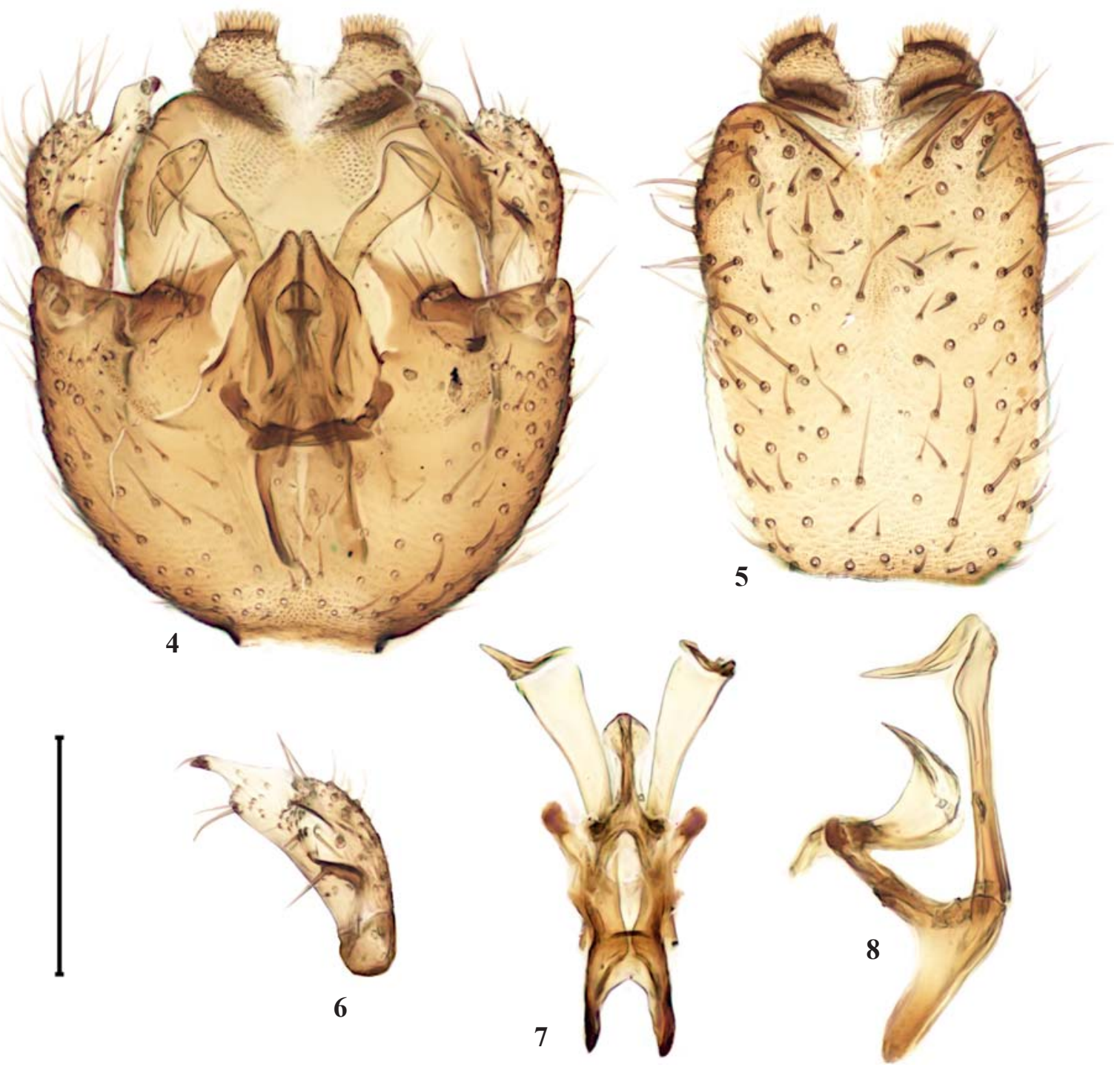

Fig. 4-8. Boletina rodentistyla, sp. n., details of male terminalia: 4 - male terminalia, ventral view; 5 - tegrite IX; 6 - gonostylus, internal view; 7 - aedeagal complex, ventral view; 8 - aedeagal complex, lateral view. Scale bar: $0.2 \mathrm{~mm}$.

Рис. 4-8. Boletina rodentistyla, sp. n., детали строения гениталий самца: 4 - гениталии самца, вентрально; 5 - тергит IX; 6 гоностиль, изнутри; 7 - комплекс эдеагуса, вентрально; 8 - комплекс эдеагуса, сбоку. Мастштаб: 0,2 мм. 


\section{Description of new species}

\section{Boletina rodentistyla Polevoi, sp.n.} Figs 4-13.

MATERIAL. Holotype. $\sigma^{7}$. Russia, Pasvik Nature Reserve, 1 $\mathrm{km} \mathrm{SE}$ of the island Varlam (Niilansaari), $80 \mathrm{~m}$ above sea level, 3.08-10.10.2007, A. Bulychev leg. (ZISP).

Paratypes. $1 \sigma^{7}$, same data as holotype (ZISP); $1 \overbrace{}^{7}$, Russia, Pasvik Nature Reserve, river Menikkajoki, $21 \mathrm{~m}$ above sea level, 14.08-25.09.2007, A. Bulychev leg. (ZISP); $1 \sigma^{7}$ Finland, Kotavaara, $190 \mathrm{~m}$ above sea level, 21.09-2.10.1998, M.Tietäväinen leg. (ZISP).

Other material. $4 \sigma^{7} \sigma^{\top}$, Russia, Krasnoyarsk region, River Pyasina, $90 \mathrm{~km} \mathrm{~N}$ of Norilsk, $70.15^{\circ} \mathrm{N}, 88.28^{\circ} \mathrm{E}, 13.08 .2015, \mathrm{M}$. Shcherbakov leg. (UTR); $5 \sigma^{\top} \sigma^{\top}$, same data, except 10.08.2015 (UTR). Also known from Finnish Lapland (J. Salmela. pers. comm.) and N Norway (J. Kjærandsen pers. comm.).

Male $(n=6)$.

Head, including face and clypeus blackish-brown, mouthparts and palpi brownish-yellow. Antenna dark-brown with brownish pubescence. Sixth flagellomere 2.40-3.55 (mean value 3.04) times longer than wide.

Thorax brown to dark-brown. Mesonotum thinly dusted with subshining longitudinal stripes along acrostichal and lateral to dorsocentral rows of setae. Laterotergite bare.

Wing length 3.67-4.16 (3.90) mm. Wings hyaline. Veins yellow except $C$ and $R$ brownish. Costa extending beyond tip of $R_{5}$ to approximately half of the distance from $R_{5}$ to $M_{l} . S c$ bare ending in costa opposite or slightly distal to the base of $R$. Sc-r located slightly distal to the middle of $S c$. The stem of $M$ fork 1.11-1.35 (1.21) times as long as $r-m$. Base of
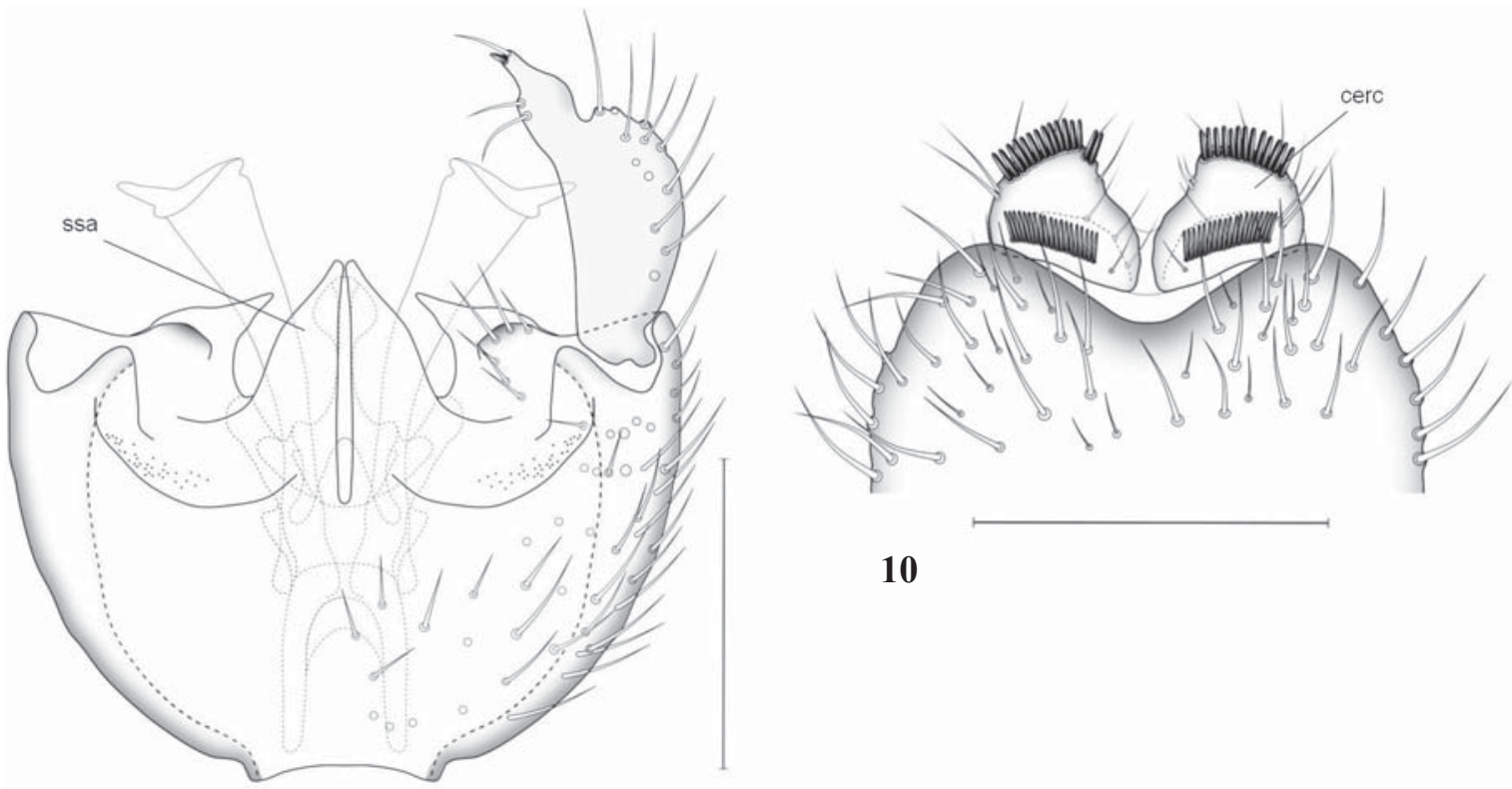

10

9
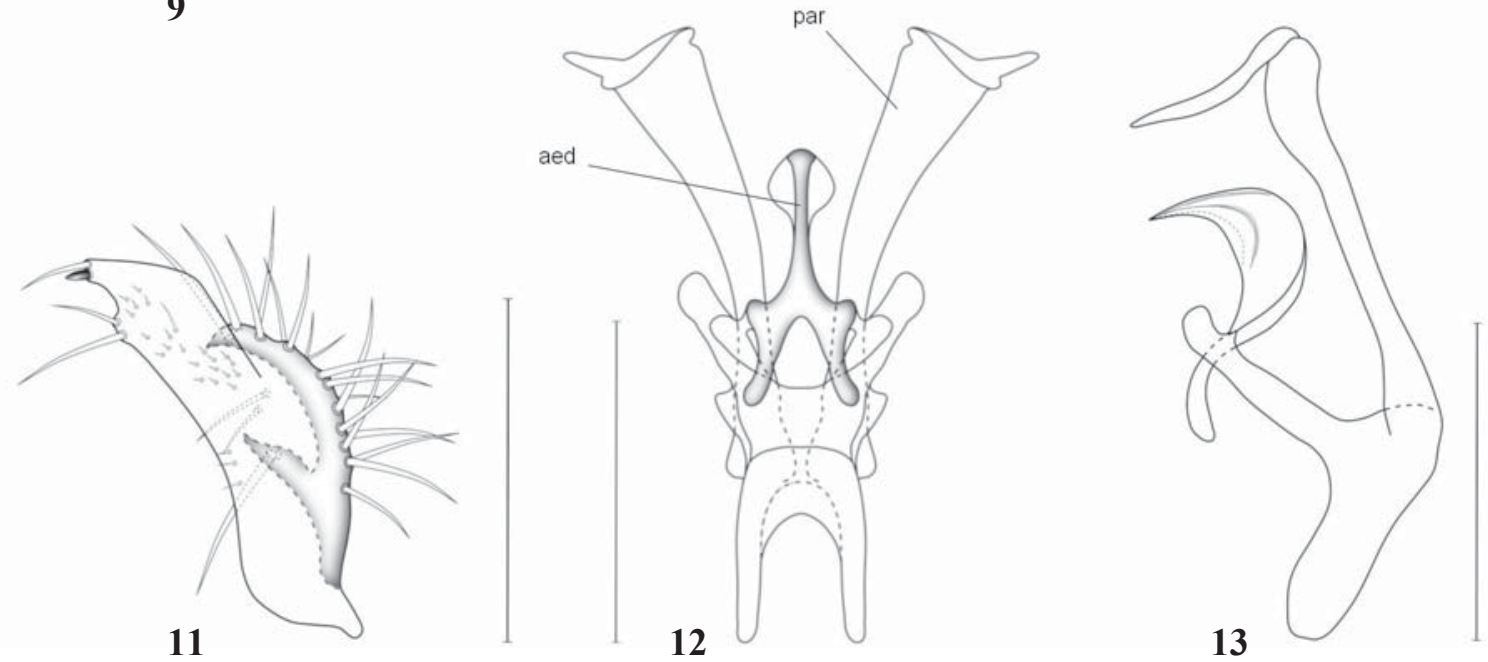

Fig. 9-13. Boletina rodentistyla, sp. n., details of male terminalia: 9 - male terminalia, ventral view (tergite IX removed); 10 - tegrite IX; 11 - gonostylus, internal view; 12 - aedeagal complex, ventral view; 13 - aedeagal complex, lateral view. Scale bars: 0.2 mm. Abbreviation: ssa - sternal submedian appendages, cerc - cercus, aed — aedeagus, par — parameres.

Рис. 9-13. Boletina rodentistyla, sp. n., детали строения гениталий самца: 9 - гениталии самца, вентрально (тергит IX не показан); 10 - тергит IX; 11 - гоностиль, изнутри; 12 - комплекс эдеагуса, вентрально; 13 - комплекс эдеагуса, сбоку. Мастштаб: 0,2 мм. Сокращения: ssa - стернальные субмедиальные выросты, cerc - церки, aed — эдеагус, par — парамеры. 
posterior $\left(M_{-}-\mathrm{Cu} A\right)$ fork slightly proximal to the base of $M$ fork. $R_{1}$ and $R_{5}$ with macrotrichia all over dorsal surface. $R-m$ bare. $M_{1}$ and $M$, bare at base. $M_{4}$ and $C u A_{1}$ with macrotrichia in the apical $1 / 2$ to $2 / 3$. Halteres yellow to brownish-yellow.

Legs yellow to yellowish-brown. Coxae yellowish-brown, sometimes mid and hind coxae or all coxa more or less strongly darkened. Trochanters brown to black. Fore tibia with 3-4 short $p v$. Mid tibia with $3 a d, 3-4 p d, 2-4 a v, 5-7 p v$. Hind tibia with 3-4 ad (+1-4 short $a d$ at the apex), 4-5 pd, $4 a v, 4 p v$. Ratio of tibia to first tarsomere for fore, mid and hind legs: 1.22-1.47 (1.36), 1.26-1.57 (1.43), 1.42-1.9 (1.71). Fore tibia with $1 p v$ spur, mid and hind tibia each with two spurs ( $a v$ spur about 3/4 as long as $p v$ spur). Tarsal claws not modified.

Abdomen dark-brown with pale hairs.

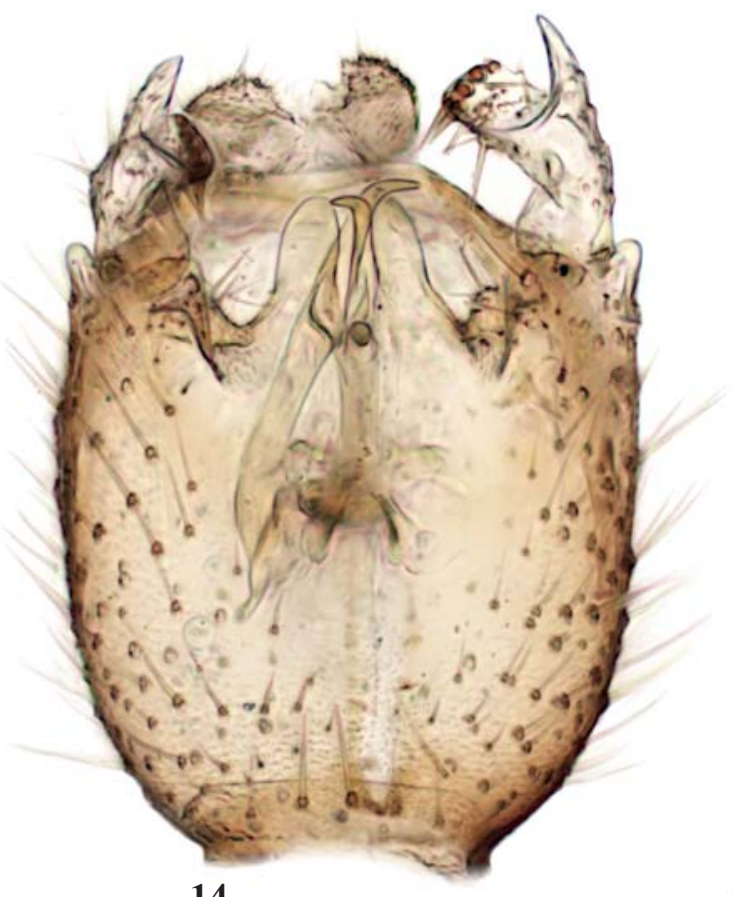

14

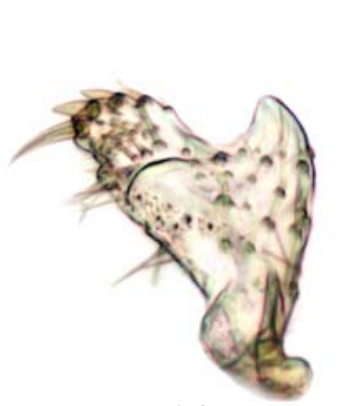

16

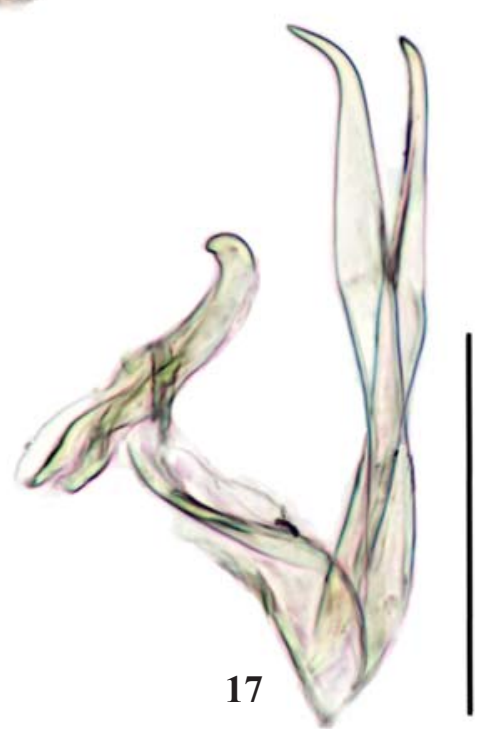

Terminalia dark-brown. Sternal submedian appendages wide basally and narrowed to the apices (Fig. 4, 9). Gonostylus of characteristic shape, resembling a grotesque animal head when viewed from the inner side (Fig. 6,11). Tergite 9 rectangular, with rather deep apical triangular depression, cercus with two combs of spines (Fig. 5, 10). Aedeagus with "cobra hood" like subapical widening, parameres rectangularly bent and widened subapically and then narrowed to apices (Fig. 7-8, 12-13).

DIAGNOSIS. A small dark Boletina with transparent wings and yellowish-brown legs (sometimes with darkened coxa). The species clearly differs from other members of the genus in the structure of the male terminalia, especially in the shape of the gonostylus and details of the aedeagal apparatus.

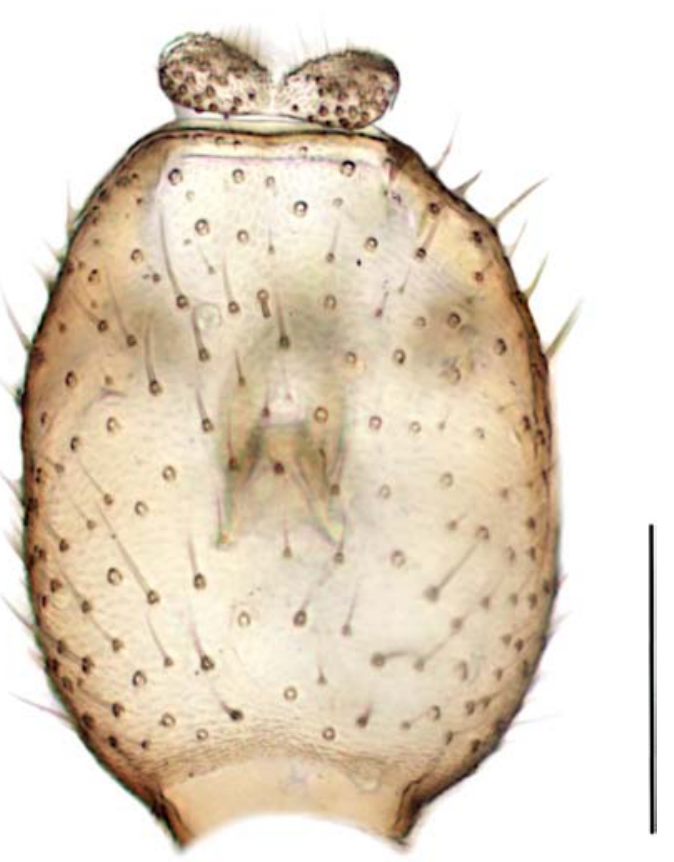

15

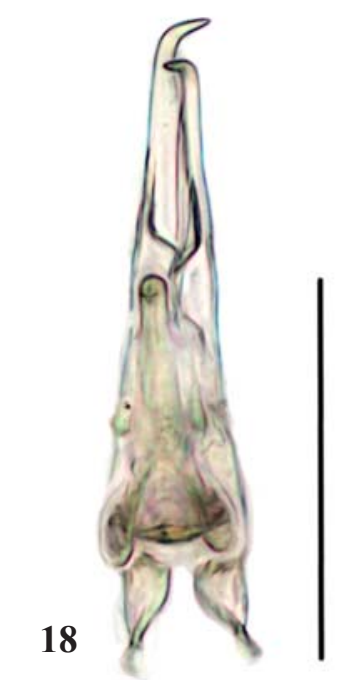

Fig. 14-18. Boletina pyasina, sp. n., details of male genitalia: 14 - male genitalia, ventral view; 15 - tegrite IX; 16 - gonostylus, internal view; 17 - aedeagal complex, ventral view; 18 - aedeagal complex, lateral view. Scale bar: $0.2 \mathrm{~mm}$.

Рис. 14-18. Boletina pyasina, sp. n., детали строения гениталий самца: 14 - гениталии самца, вентрально; 15 — тергит IX; 16 - гоностиль, изнутри; 17 - комплекс эдеагуса, вентрально; 18 - комплекс эдеагуса, сбоку. Мастштаб: 0.2 мм. 
ETYMOLOGY. The species name points to the characteristic shape of the male gonostylus, resembling a grotesque animal (e.g. rodent) head.

ECOLOGY. Specimens from Fennoscandia collected with Malaise's traps in various forest habitats. The habitats in the Taimyr peninsula are sedge-dwarf tundra communities.

DISTRIBUTION. N Palaearctic: Finland, Norway, Russia (Murmansk Province and N Siberia).
Boletina pyasina Maximova et Subbotina, sp.n.

Figs 14-24.

MATERIAL. Holotype. $\sigma^{7}$. Russia, Krasnoyarsk region, River Pyasina, $90 \mathrm{~km} \mathrm{~N}$ of Norilsk, $70.15^{\circ} \mathrm{N}, 88.28^{\circ} \mathrm{E}, 46 \mathrm{~m}$ above sea level, 13.08.2015, M. Shcherbakov leg. (UTR).

Paratypes. $2 \bigcirc^{7} \sigma^{7}$, same data as holotype (UTR); $2 \sigma^{7} \sigma^{7}$, same data as holotype (ZISP)

Other material. $12 \mathrm{O}^{7} \mathrm{O}^{7}$, same data as holotype (UTR); $2 \mathrm{O}^{\top} \sigma^{7}$,
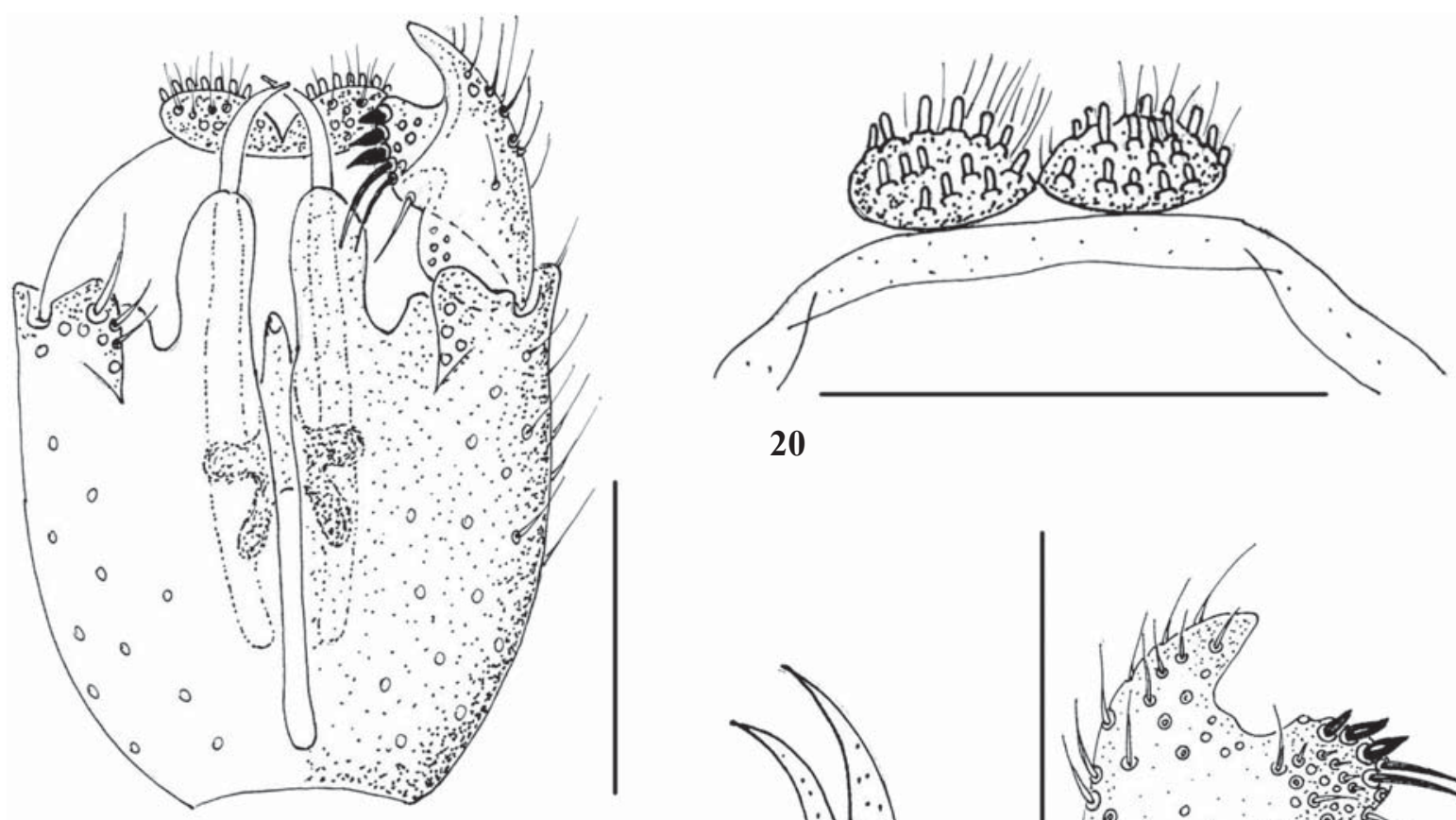

20

19
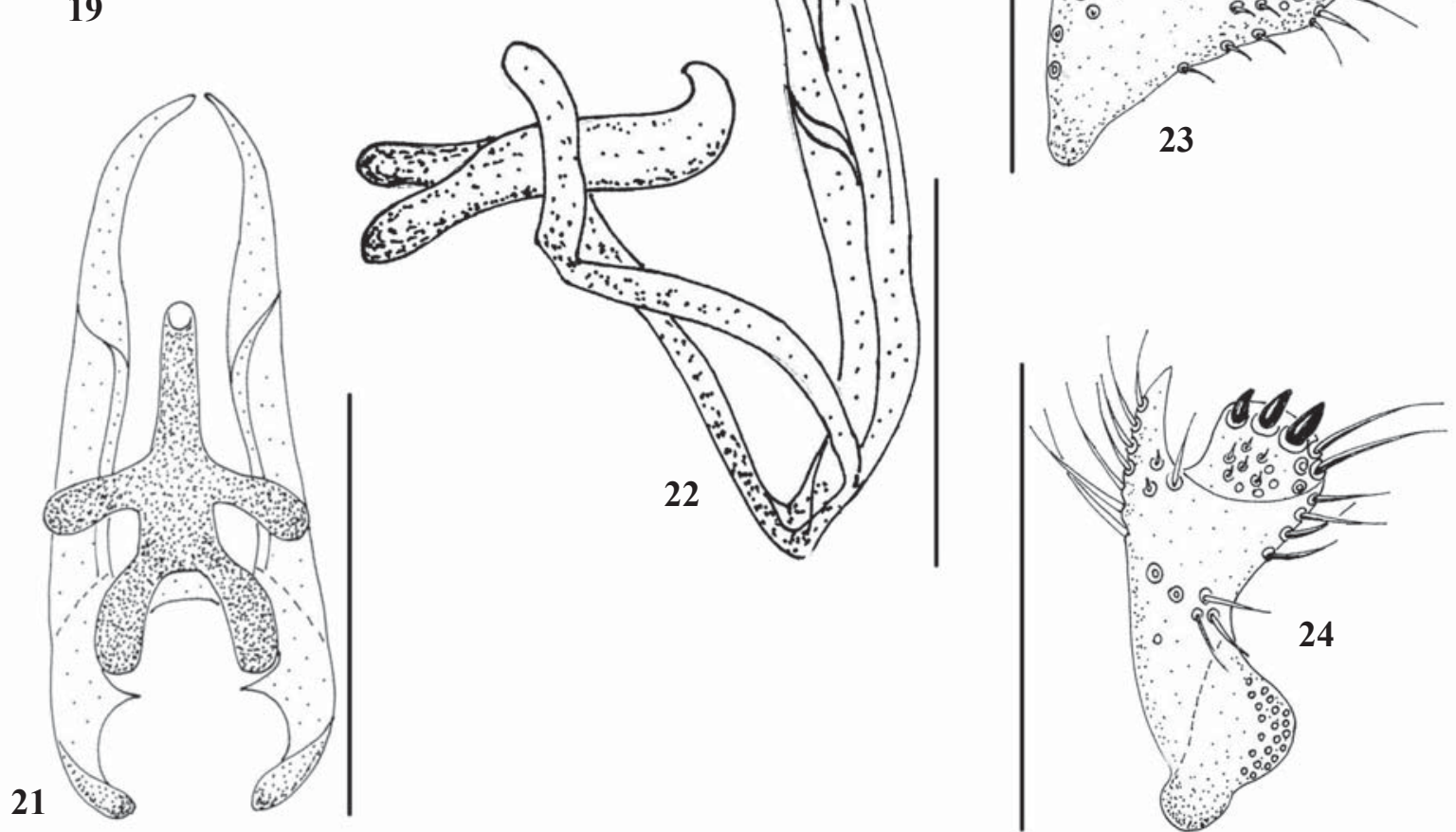

Fig. 19-24. Boletina pyasina, sp. n., details of male terminalia: 19 - male terminalia, ventral view; 20 - tegrite IX; 21 - aedeagal complex, ventral view; 22 - aedeagal complex, lateral view; 23 - gonostylus, external view; 24 - gonostylus, ventral view. Scale bar: 0.2 $\mathrm{mm}$

Рис. 19-24. Boletina pyasina, sp. n., детали строения гениталий самца: 19 — гениталии самца, вентрально; 20 - тергит IX; 21 комплекс эдеагуса, вентрально; 22 - комплекс эдеагуса, сбоку; 23 - гоностиль, с внешней стороны; 24 - гоностиль, вентрально, Мастштаб: 0,2 мм. 
Russia, Krasnoyarsk region, River Pyasina, $93 \mathrm{~km} \mathrm{~N}$ of Norilsk, $70.18^{\circ} \mathrm{N}, 88.18^{\circ} \mathrm{E}, 12.08 .2015$, M. Shcherbakov leg. (UTR).

Male $(n=5)$.

Head dark brown. Mouthparts dark brown, palpi brownish. Clypeus brown. Antenna light brown. Sixth flagellomere about twice as long as wide.

Thorax. Mesonotum dark brown, thinly dusted with subshining longitudinal stripes along acrostichal and lateral to dorsocentral rows of setae. Pleurae brown. Laterotergite bare.

Wing length 3.39-3.45 (mean value 3.42) mm. Wings hyaline. Veins yellow, except $C$ and $R$ brownish. Costa extending beyond tip of $R_{5}$ up to the middle of the distance between $R_{5}$ and $M_{l}$. Sc bare, ending in costa opposite to the base of $R_{s}$. Sc-r located slightly beyond the middle of $S c$. The stem of $M$ fork 1.1-1.2 times as long as $r-m$. Base of posterior $\left(M_{4}-\mathrm{CuA}\right)$ fork slightly beyond the base of r-m. $R_{1}$ and $R_{5}$ with macrotrichia all over dorsal surface. $R-m$ bare. $M_{1}$ and $M$ bare at the basal $1 / 3 . M_{4}$ and $C u A_{1}$ with macrotrichia in the apical $1 / 2$ to $2 / 3$. Halteres yellow.

Legs yellow. Coxa darkened basally. Trochanters brown. Fore tibia with $1 p d$ and 1-2 $p v$. Mid tibia with $3 a d, 2-3 p d$, $3 v, 3-5 a v$ and $4 p v$. Hind tibia with $3 v, 7-10 a d, 2 a v, 3-4$ $d$ and 1-3 p. Ratio of tibia to first tarsomere for fore, mid and hind legs: $1.46-1.58$ (1.53); 1.41-1.49 (1.48); 1.82-1.93 (1.88). Fore tibia with $1 p v$ spur, mid and hind tibia each with two spurs ( $a v$ spur about 0.6 times as long as $p v$ spur). Tarsal claws slightly thickened.

Abdomen brownish, with pale hairs.

Terminalia brown. Gonocoxites divided by deep median cleft, sternal submedian appendages relatively long, apically rounded (Fig. 14, 19). Gonostylus bears 3 short black spines and 2-3 longer seta apically, and with the well-developed horn-like ventral process (Fig. 16, 23-24). Tergite 9 ovate, cerci rounded, covered with irregularly arranged 13-18 spines (Fig. 15, 20). Parameres relatively narrow, slightly bent near the apex and then pointed (Fig. 17-18, 21-22).

Female unknown.

DIAGNOSIS. A small dark Boletina with transparent wings and yellow legs. B. pyasina is most similar to B. silvatica Dziedzicki, 1885, which also has gonostylus with the ventral horn-like process. These species can be easily distinguished by sternal submedian appendages of gonocoxites, which are relatively wide and rounded apically in B. pyasina, and pointed in $B$. sylvatica. Other related species $B$. triangularis Polevoi, 1995, B. subtriangularis Polevoi et Hedmark, 2004 have a shorter ventral process on the gonostylus and short triangular sternal submedian appendages of gonocoxites. Boletina nuortti Salmela, 2016 is also somewhat similar, but lacking ventral process on the gonostylus and has distinctly shorter parameres as compared to all above-mentioned species.

ETYMOLOGY. The species name is derived from the River Pyasina in the Krasnoyarsk Region of Russia.

ECOLOGY. The habitats in the type locality are sedgedwarf tundra communities. The species has been collected with Malaise traps and sweep net.

DISTRIBUTION. N Palaearctic: Russia (N Siberia).

\section{Conclusion}

With less than 80 known species, the fungus gnats fauna of the Taimyr Peninsula can't be considered as sufficiently studied. It is however characterized by some traits similar to other local northern faunas, e.g. a significant ratio of widely distributed (Holarctic and Transpalaearctic) species and presence of certain species endemic to high latitude arctic and subarctic regions. A more detailed discussion on the biogeographical structure of the fauna will be possible when more materials are available from the area.

Acknowledgements. We are grateful to Olga Makarova (Moscow), Anatoli Barkalov (Novosibirsk), and Mikhail Shcherbakov (Tomsk) for giving us the possibility to study their materials and for the information on the collecting localities. The work of A. Polevoi was carried out under state order implemented by Karelian Research Centre RAS (Forest Research Institute).

Competing interests. The authors declare no competing interests.

\section{References}

Amorim D.D.E.S., Rindal E. 2007. Phylogeny of the Mycetophiliformia, with proposal of the subfamilies Heterotrichinae, Ohakuneinae, and Chiletrichinae for the Rangomaramidae (Diptera, Bibionomorpha) // Zootaxa. Vol.1535. P.1-92.

Borkent C.J., Wheeler T.A. 2012. Systematics and Phylogeny of Leptomorphus Curtis (Diptera: Mycetophilidae) // Zootaxa. Vol.3549. No.1. P.1-117.

Chandler P.J. 1993. The Holarctic species of the Mycetophila fungorum (De Geer) group (Diptera, Mycetophilidae) // British Journal of Entomology and Natural History. Vol.6. P.5-11.

Chandler P.J. 1994. Rymosia Winnertz (Diptera: Mycetophilidae), a newly recognised element of wetland faunas, with five species new to Britain and a key to species // Entomologist's Gazette. Vol.45. P.199-220.

Chandler P.J. 2001. Fungus gnats (Diptera: Sciaroidea) new to Britain // British Journal of Entomology and Natural History. Vol.13. P.215-243.

Gagné R.J. 1981. A monograph of Trichonta with a model for the distribution of Holarctic Mycetophilidae (Diptera) // United States Department of Agriculture Technical Bulletin. Vol.1638. P.1-64.

Holmgren A.E. 1883. Insecta a viris doctissimus Nordenskiöld illum ducem sequentibus in insulis Waigatsch et Novaja Semlia anno 1875 collecta: Diptera // Entomologisk Tidskrift. Vol.4. P.162-190.

Jakovlev J., Salmela J., Polevoi A., Penttinen J., Vartija N.-A. 2014. Recent noteworthy findings of fungus gnats from Finland and northwestern Russia (Diptera: Ditomyiidae, Keroplatidae, Bolitophilidae and Mycetophilidae) // Biodiversity Data Journal. Vol.2. No.e1068. P.1-139.

Jürgenstein S., Kurina O., Põldmaa K. 2015. The Mycetophila ruficollis Meigen (Diptera, Mycetophilidae) group in Europe: elucidating species delimitation with COI and ITS2 sequence data // ZooKeys. Vol.508. P.15-51.

Kjærandsen J. 2006. Review of fungus gnats in the genus Tarnania Tuomikoski, with a phylogeny of the Rymosia s.l. genus group (Diptera/: Mycetophilidae) // Insect Systematics \& Evolution. Vol.37. No.2. P.121-148.

Kjærandsen J., Jordal J.B. 2007. Fungus gnats (Diptera: Bolitophilidae, Diadocidiidae, Ditomyiidae, Keroplatidae and Mycetophilidae) from Møre og Romsdal // Norwegian Journal of Entomology. Vol.54. P.147-171.

Kjærandsen J., Hedmark K., Kurina O., Polevoi A., Økland B., Götmark F. 2007a. Annotated checklist of fungus gnats from Sweden (Diptera: Bolitophilidae, Diadocidiidae, Ditomyiidae, Keroplatidae and Mycetophilidae) // Insect Systematics \& Evolution, Supplement. Vol.65. P.1-165.

Kjærandsen J., Kurina O., Ólafsson E. 2007b. The fungus gnats of Iceland (Diptera, Keroplatidae \& Mycetophilidae) // Insect Systematics \& Evolution, Supplement. Vol.64. P.61-96.

Kjærandsen J., Martinsson S., Hedmark K., Evenhuis N.L. 2009. On the genus Urytalpa Edwards (Diptera: Keroplatidae) in the Nordic and Nearctic regions, with fixation of a new type species and a key to world males // Zootaxa. Vol.50. No.2160. P.29-50.

Krivosheina N.P., Zaitzev A.I., Jakovlev E.B. 1986. [Insects destroying the fungi in the forests of European part of Russia]. Moscow: Nauka. 309 pp. [In Russian] 
Laffoon J. 1956. A revision of the Nearctic species of Fungivora (Diptera, Mycetophilidae) // Iowa State College Journal of Science. Vol.31. No.2. P.141-340.

Laštovka P. 1972. Holarctic species of Mycetophila rufficollisgroup (Diptera, Mycetophilidae) // Acta entomologica bohemoslovaca. Vol.69. P.275-294.

Lundström C. 1915. Diptera Nematocera aus den arctischen Gegenden Sibiriens // Mémoires de l'Académie Impériale des Sciences. VIIIe serie. Classe Physico-Mathématique. Vol.29. No.8. P.1-33.

Martinsson S., Kjærandsen J. 2012. Katatopygia gen. n., a monophyletic branch segregated from Boletina (Diptera, Mycetophil idae) // ZooKeys. Vol.175. P.37-67.

Ostroverkhova G.P., Maksimova Yu. V. 2000. A preliminary list of the fungus gnats (Diptera, Sciaroidea, excluding Sciaridae) from Kuznetsk Alatau Mountains // International Journal of Dipterological Reasearch. Vol.11. No.3. P.145-155.

Polevoi A.V. 2000. [Fungus gnats (Diptera: Bolitophilidae, Ditomyiidae, Keroplatidae, Diadocidiidae, Mycetophilidae) of Karelia]. Petrozavodsk: Karelian Research Centre of Russian Academy of Sciences. 84 pp. [In Russian, with English summary]

Polevoi A.V. 2010. [Fungus gnats (Diptera: Bolitophilidae, Keroplatidae, Mycetophilidae) of Pasvik strict nature reserve] // Trudy Karel'skogo nauchnogo tsentra RAN. Seriya biogeographiya. No.1. P.95-104. [in Russian, with English summary].

Polevoi A.V., Barkalov A.V. 2017. Fungus gnats (Diptera: Bolitophilidae, Diadocidiidae, Keroplatidae, Mycetophilidae) of the lower course of Anadyr River, Chukotskii Autonomnyi Okrug, Russia // Eurasian Entomological Journal. Vol.16. No.2. P.119-128.

Saigusa T. 2006. Homology of wing venation of Diptera. Fukuoka: Privately published by the author. $26 \mathrm{pp}$

Salmela J., Suuronen A., Kaunisto K. 2016. New and poorly known Holarctic species of Boletina Staeger, 1840 (Diptera, Mycetophilidae) // Biodiversity Data Journal. Vol.4. No.e7218. P.1-28.

Sasakawa M., Kimura T. 1974. Japanese Mycetophilidae (Diptera). 7. Genus Boletina Staeger// The Scientific reports of Kyoto Prefectural University. Agriculture. Vol.26. P.44-66.

Söli G.E.E. 1997a. The adult morphology of Mycetophilidae (s. str.) with a tentative phylogeny of the family (Diptera, Sciaroidea) // Entomologica Scandinavica Supplement. Vol.50. P.5-55.

Söli G.E.E. 1997b. The systematics and phylogeny of Coelosia Winnertz, 1863 (Diptera, Mycetophilidae)//Entomologica Scandinavica Supplement. Vol.50. P.57-134.
Söli G., Rindal E., Gammelmo Ø. 2015. Mycetophilidae, Bolitophilidae \& Keroplatidae (Fungus gnats) // Böcher J., Kristensen N.P., Pape T., Wilhelmsen L. (Eds.). The Greenland Entomofauna: an Identification Manual of Insects, Spiders and Their Allies. Leiden-Boston: Brill. P.256-269.

Subbotina E.Yu. 2014. Fungus gnats (Diptera: Bolitophilidae, Keroplatidae, Mycetophilidae) of subtaiga zone of West Siberia // Euroasian Entomological Journal. Vol.13. No.1. P.47-58.

Väisänen R. 1984. A monograph of the genus Mycomya Rondani in the Holarctic region (Diptera, Mycetophilidae) // Acta Zoologica Fennica. Vol.177. P.1-346.

Zaitzev A.I. 1982. [Holarctic fungus gnats of the genus Sciophila Meig. (Diptera, Mycetophilidae)]. Moscow: Nauka. 75 pp. [In Russian]

Zaitzev A.I. 1983. [A review of Holarctic species of the subgenus Allodia s.str. (Diptera, Mycetophilidae)] // Zoologicheskiy zhurnal. Vol.62. No.12. P.1915-1920 [in Russian, with English summary].

Zaitzev A.I. 1985. [ Holarctic species of the fungus gnats of the genus Brevicornu group fissicauda and proximum (Diptera, Mycetophilidae)] // Vestnik Zoologii. No.5. P.40-47 [in Russian, with English summary].

Zaitzev A.I. 1988. [Fungus gnats of the Sericoma, Griseicolle and Ruficorne species groups of the genus Brevicornu Marshall (Diptera, Mycetophilidae) of Holarctic fauna] // Entomologicheskoe obozrenie. Vol.67. No.2. P.391-404 [in Russian, with English summary].

Zaitzev A.I. 1994. [Fungus gnats of the fauna of Russia and adjacent regions. Part 1.]. Moscow: Nauka. 288 pp. [In Russian, with English summary]

Zaitzev A.I. 1999. [Families Bolitophilidae, Diadocidiidae, Ditomyiidae, Keroplatidae, Mycetophilidae]//Sidorenko V.S., Kupyanskaya A.N., Leley A.S., Nemkov P.G., Kholin S.K. (Eds.). Opredelitel' nasekomykh Dal'nego Vostoka Rossii. Tom.VI. Diptera i Siphonaptera. Ch.1. Vladivostok: Dal'nauka. P.134239 [in Russian].

Zaitzev A.I. 2003. Fungus gnats (Diptera, Sciaroidea) of the fauna of Russia and adjacent regions. Part 2. // International Journal of Dipterological Reasearch. Vol.14. No.2-4. P.77-386.

Zaitzev A.I., Polevoi A. V. 2001. Holarctic species of the Boletina erythropyga group (Diptera, Mycetophilidae) // Studia dipterologica. Vol.8. No.2. P.639-644. 